\title{
Unusual Etiologies for Vascular Duodenal Compression Mimicking the Superior Mesenteric Artery (SMA) Syndrome: The SMA-Like Syndrome
}

\author{
Peter E. Dross ${ }^{1}$ Shawdon Molavi ${ }^{1} \quad$ Alex Chan $^{1} \quad$ Rachael Latshaw $^{1} \quad$ Pankaj Chhabra ${ }^{1}$ \\ 1Department of Radiology, Christiana Care Health System, Newark, \\ Address for correspondence Peter E. Dross, MD, Department of \\ Delaware, United States \\ Radiology, Christiana Care Health System, 4755 Ogletown-Stanton Road, \\ Newark, DE 19718, United States (e-mail: PDross@christianacare.org).
}

J Gastrointestinal Abdominal Radiol ISGAR 2019;2:140-146

\begin{abstract}
We report a series of nine cases of unusual etiologies for vascular duodenal compresKeywords

- superior mesentery artery syndrome

- SMA-like syndrome

- duodenal compression

- vascular compression sion that do not meet the diagnostic criteria for superior mesenteric artery (SMA) syndrome and propose that "SMA-like syndrome" be used to classify these vascular duodenal compression cases.
\end{abstract}

\section{Introduction}

Superior mesenteric artery syndrome (SMAS) was originally described by Rokitansky in $1861^{1}$ and later described as a chronic duodenal ileus by Wilkie in $1927^{2}$ whose name is occasionally used eponymously. SMAS consists of obstruction of the third portion of the duodenum due to compression between the superior mesenteric artery (SMA) and the aorta. Traditional imaging method for establishing the diagnosis of SMAS was an upper gastrointestinal barium (UGI) study. ${ }^{3}$ Computed tomography (CT) is considered the diagnostic test of choice as it allows for the evaluation of the mesoaortic vascular anatomy, duodenal compression, and proximal dilatation simultaneously. ${ }^{4}$ The aortomesenteric angle (AMA) and aortomesenteric distance (AMD) in patients with SMAS have been reported to be 6 to 22 degrees and 2 to $8 \mathrm{~mm}$, respectively. ${ }^{4-7}$ However, other vascular structures in the abdomen and pelvis may cause compression of adjacent hollow viscera.

Lamba et $\mathrm{al}^{8}$ refer to a heterogeneous group of disorders including the median arcuate ligament syndrome (MALS), May-Thurner syndrome, nutcracker syndrome, SMAS, ureteropelvic junction obstruction, and ovarian vein syndrome (OVS) as "vascular compression syndromes."

We report a series of nine cases, all presenting with third portion duodenal obstruction secondary to $\mathrm{CT}$ proven vascular compression, none of whom met the strict diagnostic criteria for SMAS. We propose the term "SMA-like syndrome" for patients presenting with third portion duodenal obstruction caused by vascular duodenal compression other than the SMA/aorta of SMAS.

\section{Patients and Methods}

This retrospective study included nine patients who presented to our institution with postprandial epigastric pain, abdominal distension, nausea and vomiting, and imaging evidence (UGI and/or CT) of transverse (third portion) duodenal obstruction between January 2016 and January 2019.

All patients underwent CT evaluation on a Siemens Somatom (Erlangen, Germany) multidetector CT scanner utilizing our contrast enhanced routine abdomen and pelvis protocol. Axial 3-mm thick sections CT images from the lung bases to the pubic ramus were acquired 65 seconds following the administration of Omnipaque 350 (General Electric Company, MA, United States) intravenous contrast, dosed at $1.5 \mathrm{~mL} / \mathrm{kg}$, injected at a rate of $3 \mathrm{~mL} / \mathrm{s}$ during the portal venous phase. Images were also reconstructed utilizing multiplanar reformatted images in the sagittal and coronal planes at $3 \mathrm{~mm}$ thickness, utilizing high and low frequency reconstruction kernels, respectively.

The AMA and AMD were measured for each case (-Table 1). The AMA was measured on sagittal reformatted received

June 12, 2019

accepted after revision

June 16, 2019

published online

August 22, 2019
DOI https://doi.org/

10.1055/s-0039-1694833

ISSN 2581-9933.
(C)2019 Indian Society of Gastrointestinal and Abdominal Radiology
License terms

(우 (1) $\odot \circledast$ 
Table 1 Measured aorto-mesenteric angle and aorto-mesenteric distance in symptomatic patient population

\begin{tabular}{|l|l|l|}
\hline Patient & AMA (degrees) & AMD (mm) \\
\hline 1 & 48 & 25 \\
\hline 2 & 20 & 5 \\
\hline 3 & 54 & 27 \\
\hline 4 & 16 & 7 \\
\hline 5 & 32 & 20 \\
\hline 6 & 29 & 5.5 \\
\hline 7 & 26 & 10 \\
\hline 8 & 33 & 7.5 \\
\hline 9 & 11 & 4 \\
\hline
\end{tabular}

Abbreviations: AMA: aorto-mesenteric angle; AMD: aorto-mesenteric distance.

images and the AMD measured between the anterior margin of the aorta and the posterior aspect of the SMA at the level of the duodenal (third portion) crossing. Any patient with duodenal compression between the SMA and aorta and meeting the reported SMAS standards for AMA (6-22 degrees) and AMD (2-8 $\mathrm{mm})^{4-8}$ was excluded from this study.

\section{Results}

The nine patients in this series were comprised of five males and four females with age ranging from 20 to 88 years at the time of presentation. All patients presented with abdominal distention, postprandial abdominal pain, and nausea and vomiting. Four patients were initially evaluated by formal barium meal studies (UGI) all of which showed gastric and duodenal dilatation with an obstruction at the third part of the duodenum (-Figs. 1A, 2A, 3A, and 4A). Definitive diagnosis was made by CT scan with IV contrast in all nine patients.

CT of patient 1 in this series showed a highly unusual duodenal compression between the right gonadal vein and the ileocolic vein (-Figs. 1B, 1C, and 5) and had an AMA of
48 degrees and an AMD of $25 \mathrm{~mm}$. Patient 2 showed duodenal compression between the jenunal branch of the SMA and the distal abdominal aorta (-Fig. 2B and C) and had an AMA of 20 degrees and an AMD of $5 \mathrm{~mm}$. Patient 3 demonstrated duodenal compression between the jejunal branch of the SMA and the abdominal aortic aneurism (AAA; - Fig. 3B and $\mathbf{C}$ ) with an AMA of 54 degrees and an AMD of $27 \mathrm{~mm}$. Patient 4 showed duodenal compression between the SMA/ SMV and a retroperitoneal cyst (-Fig. 6) with an AMA of 16 degrees and an AMD of $7 \mathrm{~mm}$. Patient 5 had a prior resection of a retroperitoneal sarcoma and a left hemicolectomy and duodenal compression between the superior mesenteric vein (SMV) and aorta (-Fig. 4B) with an AMA of 32 degrees and an AMD of $20 \mathrm{~mm}$. Patient 6 exhibited duodenal compression between the distal SMA, just distal to the takeoff of the jejunal branch, and the left common iliac artery ( - Fig. 7) with an AMA and AMD of 29 degrees and $6 \mathrm{~mm}$, respectively. Patient 7 demonstrated a duodenal compression between the SMV and the IVC ( - Fig. 8) with an AMA of 26 degrees and an AMD of $10 \mathrm{~mm}$. Patient 8 exhibited duodenal compression between distal branches of the SMV and the IVC ( - Fig. 9) with an AMA of 33 degrees and an AMD of $8 \mathrm{~mm}$. Patient 9 had duodenal compression between the SMV and IVC (-Fig. 10) with an AMA of 11 degrees and an AMD of $4 \mathrm{~mm}^{9}$

\section{Discussion}

The incidence of SMAS in the general population has been reported by Lee et $\mathrm{al}^{10}$ to be between 0.0024 and $0.34 \%$ and by Anderson et $\mathrm{al}^{11}$ at $0.2 \%$. Two-thirds of patients with SMAS are between 10 and 39 years of age ${ }^{12}$ with females more commonly affected. Because of the erect posture of humans, the SMA exits the abdominal aorta at an acute downward angle (-Fig. 11) that the third part of the duodenum, suspended by the ligament of Treitz, passes through and thus is vulnerable to becoming pinched between the SMA anteriorly and the aorta and vertebral column posteriorly. The duodenum crosses the vertebral column in most people at the L3 level. ${ }^{13}$ A short suspensory ligament can bring the duodenum higher

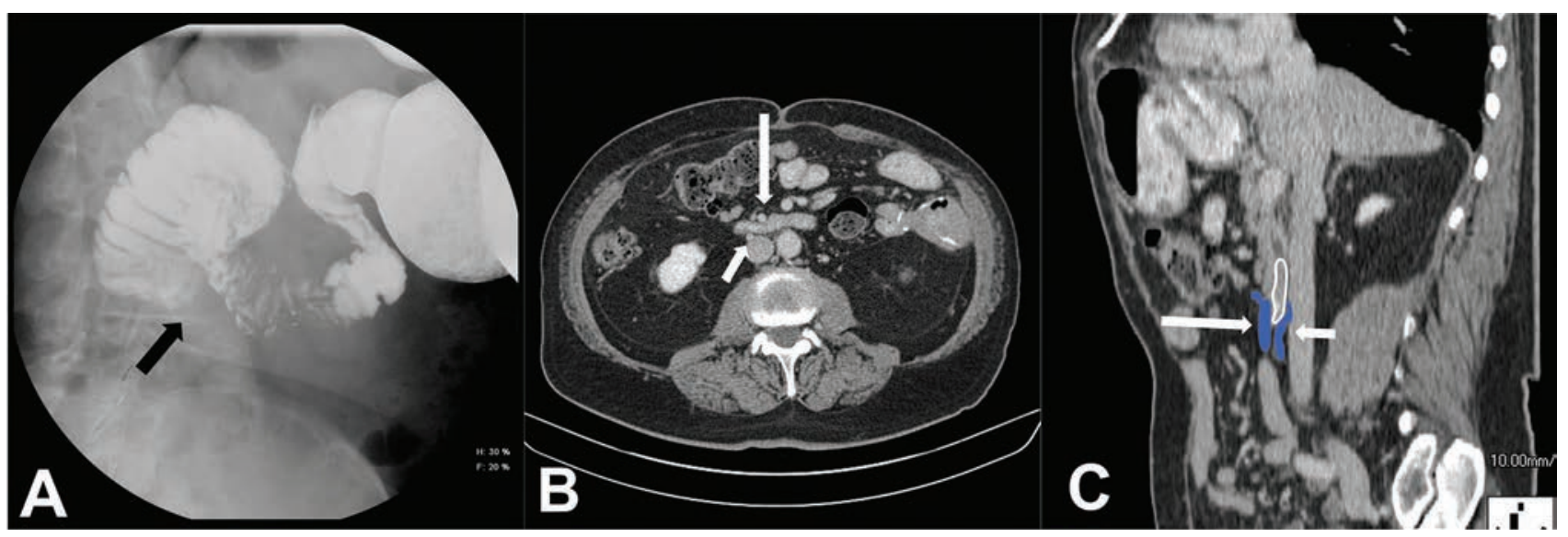

Fig. 1 Superior mesenteric artery-like syndrome due to compression by the ileocolic vein and the right gonadal vein. (A) Upper gastrointestinal (UGI) study demonstrating focal narrowing at the third portion of the duodenum (black arrow). (B) Corresponding CT demonstrates source of compression as the right gonadal vein (short arrow) and the ileocolic vein (long arrow). (C) Sagittal oblique CT reconstruction also showing the right gonadal vein (blue overlay, short arrow) and the ileocolic vein (blue overlay, long arrow) compressing the duodenum (white outline). $\mathrm{CT}$, computed tomography. 


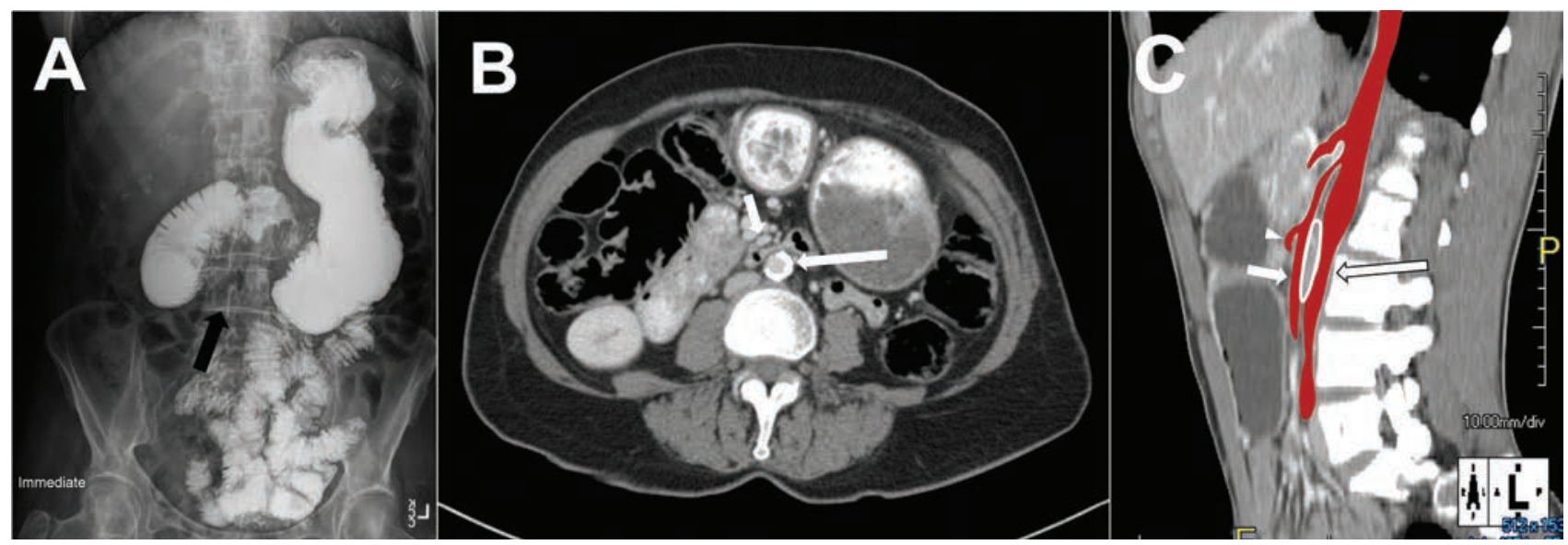

Fig. 2 SMA-like syndrome due to compression by the jejunal branch of the SMA and the distal aorta. (A) Upper GI study demonstrating focal narrowing at the third portion of the duodenum (black arrow). (B) Corresponding CT demonstrates source of compression as the jejunal branch of the SMA (short arrow) and the aorta (long arrow). (C) Sagittal CT reconstruction shows the aorta, celiac artery, and the SMA with multiple branches (red overlay). The origin of the right colic artery is seen just proximal to the point of compression (white arrow head). The duodenum (white outline) is compressed between the jejunal branch of the SMA (short arrow) and the distal aorta (long arrow). SMA, superior mesenteric artery; $\mathrm{CT}$, computed tomography; $\mathrm{Gl}$, gastrointestinal.

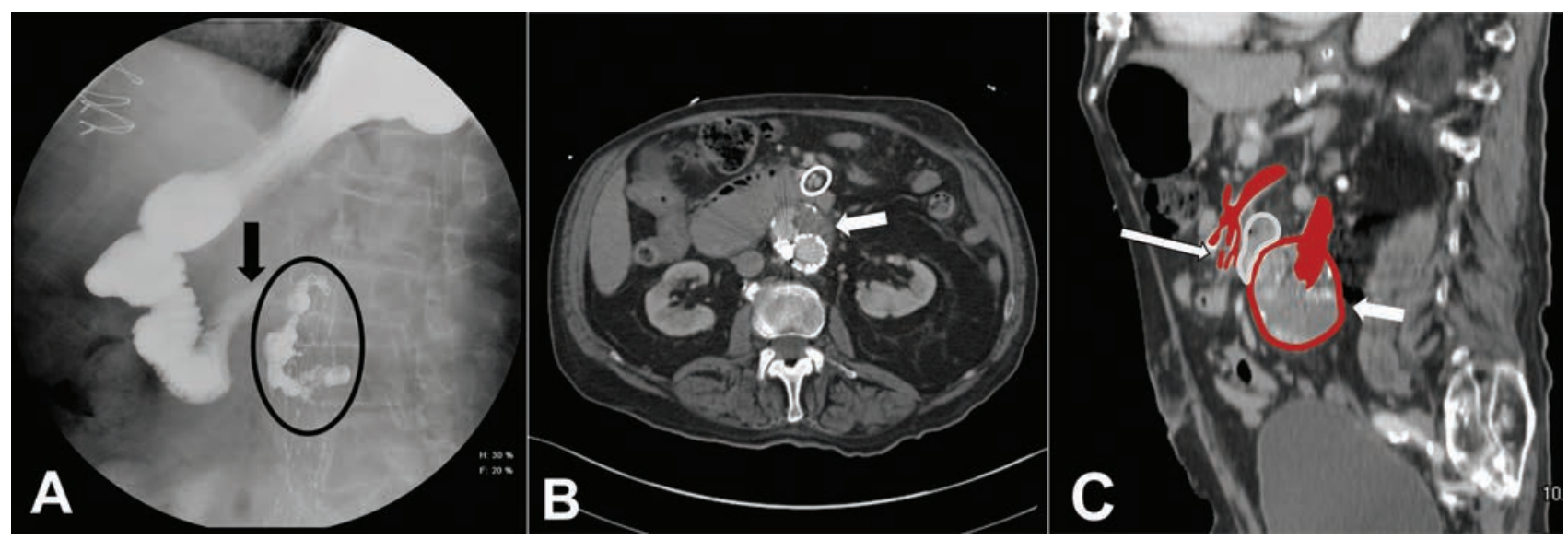

Fig. 3 SMA-like syndrome due to compression by jejunal branches of the SMA and an abdominal aortic aneurysm. (A) Upper GI study demonstrating focal narrowing at the third portion of the duodenum (black arrow). Note the adjacent embolization material (black circle) and presence an endovascular aortic graft. (B) Corresponding CT demonstrates source of compression as jejunal branches of the SMA (white circle) and an abdominal aortic aneurysm (white arrow). (C) Sagittal reconstructions shows compression of the duodenum (white outline) by jejunal branches of the SMA (long arrow) and an abdominal aortic aneurysm sac (red outline, short arrow). Red overlay designates the jejunal arteries and the aorta. SMA, superior mesenteric artery; CT, computed tomography; GI, gastrointestinal.
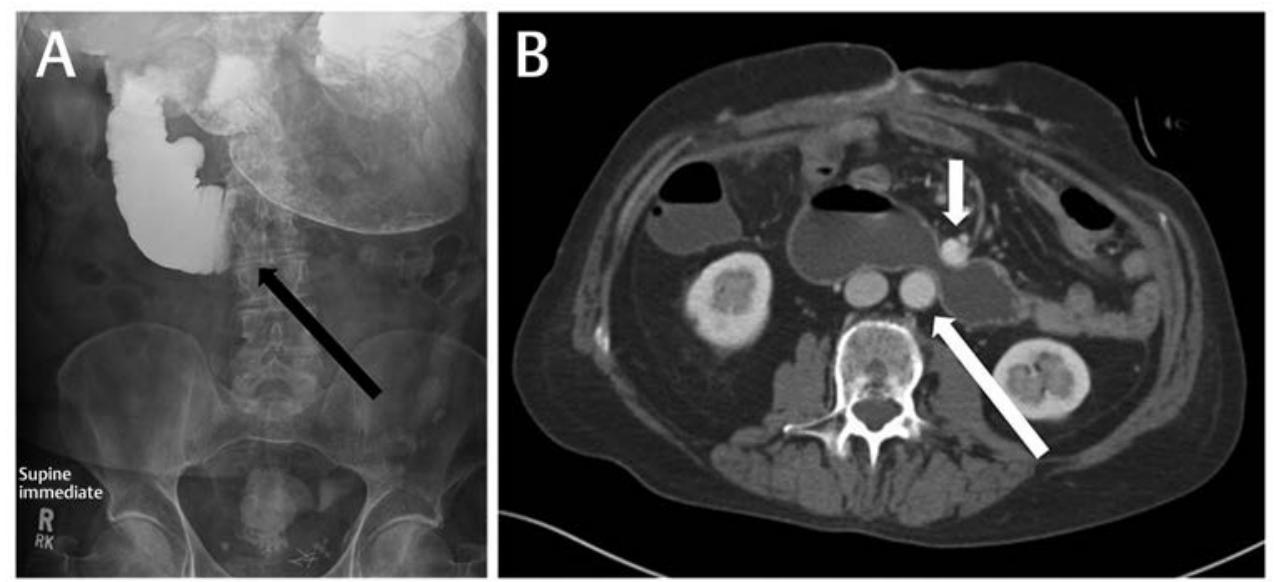

Fig. 4 SMA-like syndrome due to compression by the SMV and the aorta. (A) Upper Gl study demonstrating focal narrowing at the third portion of the duodenum (black arrow). (B) Corresponding CT demonstrates source of compression as the SMV (short arrow) and the aorta (long arrow). GI, gastrointestinal; SMA, superior mesenteric artery; SMV, superior mesenteric vein. 
(i.e., shortens the AMD) into the vascular angle and more vulnerable to compression.

Ahmed et $\mathrm{al}^{13}$ state that SMAS should be distinguished from SMA-like syndrome in which the dilatation of the duodenum is a part of a generalized duodenal dilatation (megaduodenum) of variable etiology and not caused by a compressing structure. Gondos ${ }^{14}$ studied 11 cases of systemic sclerosis in which all patients were found to have

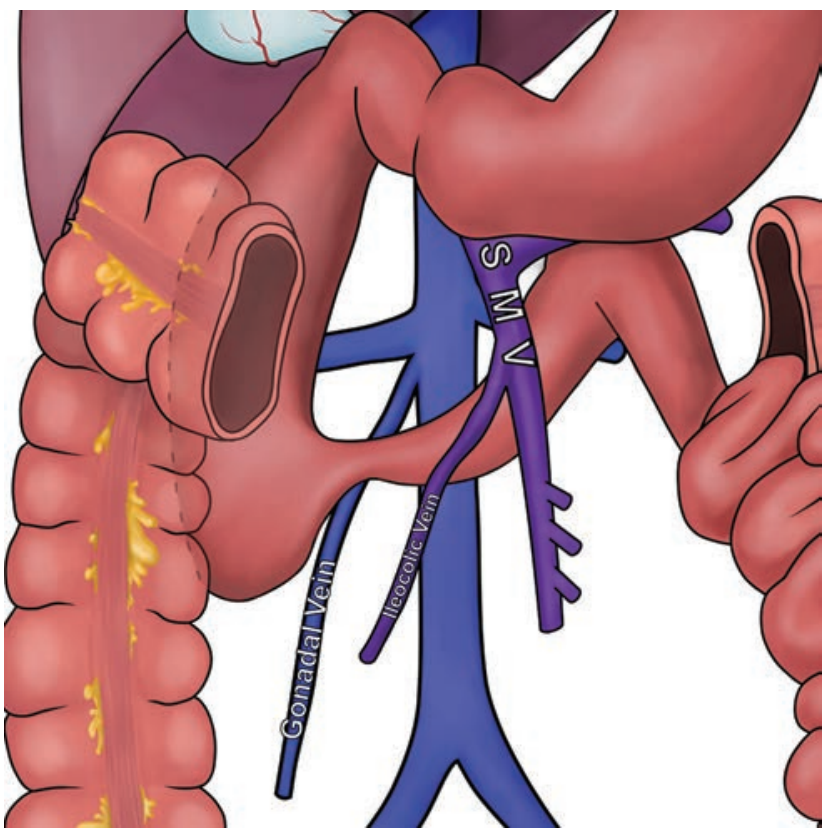

Fig. 5 Diagram of SMA-like syndrome due to compression by the ileocolic vein and the right gonadal vein. SMA, superior mesenteric artery. atrophy of the muscle layers of the duodenum and replacement by collagenous tissue leading to diminished muscle tone and diminished peristalsis and duodenal dilatation. The duodenal compression (from SMA imprinting) in these cases occurs only secondarily as a response to dilatation and loss of muscle tone of the duodenum. There are many pathologic conditions that may predispose to the development of a megaduodenum and, in turn, an imprinting of the duodenum by the SMA and include scleroderma, dermatomyositis, lupus erythematosus, diabetes, amyloidosis, and chronic idiopathic intestinal pseudo-obstruction. ${ }^{15}$

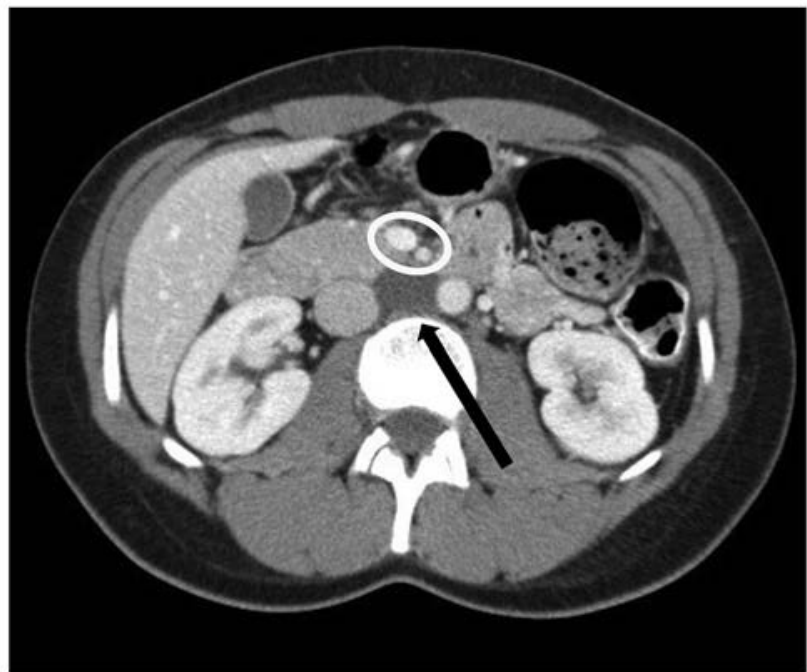

Fig. 6 SMA-like syndrome due to compression by the SMA and SMV (white circle) and a retroperitoneal cyst (black arrow). SMA, superior mesenteric artery; SMV, superior mesenteric vein.

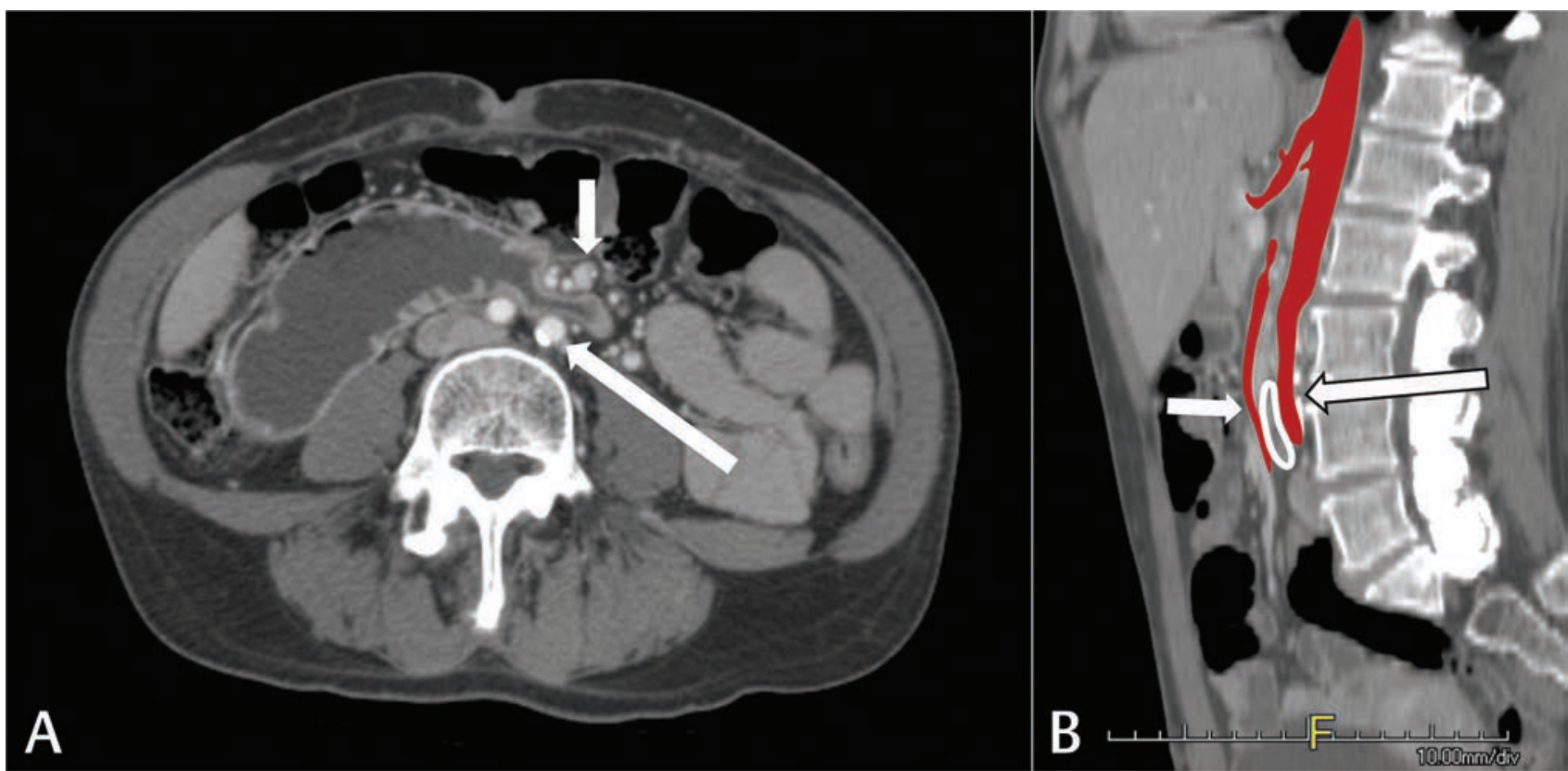

Fig. 7 (A) Axial CT showing SMA-like syndrome due to compression by the distal SMA just distal to the origin of the jejunal branch (short arrow) and the left common iliac artery (long arrow). (B) Sagittal CT reconstruction demonstrating the aorta, celiac artery and SMA (red overlay). The duodenum (white outline) is compressed by the distal SMA (short arrow) and the left common iliac artery (long arrow). SMA, superior mesenteric artery; $\mathrm{CT}$, computed tomography. 


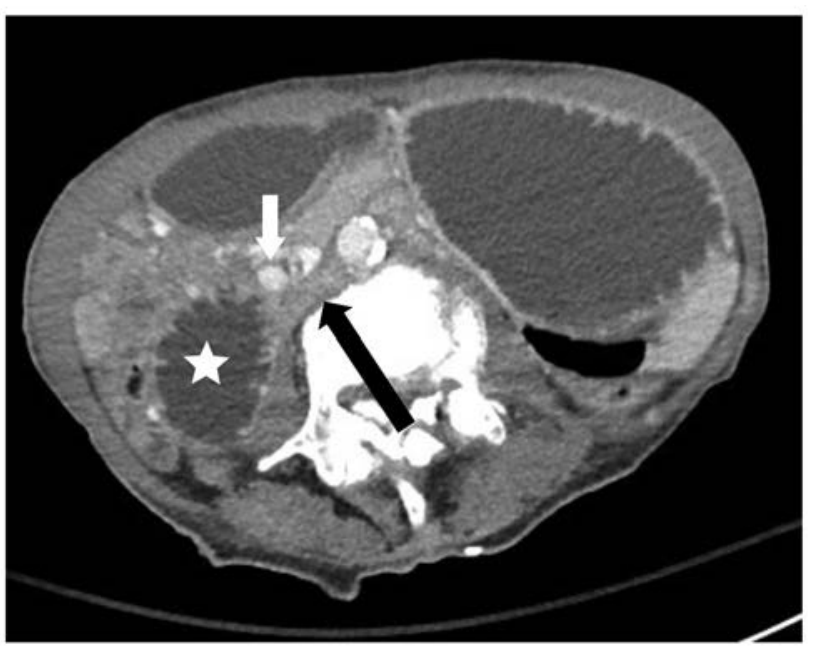

Fig. 8 SMA-like syndrome with duodenal obstruction (white star) due to compression by the SMV (white arrow) and the collapsed IVC (black arrow). IVC, inferior vena cava; SMA, superior mesenteric artery; SMV, superior mesenteric vein.

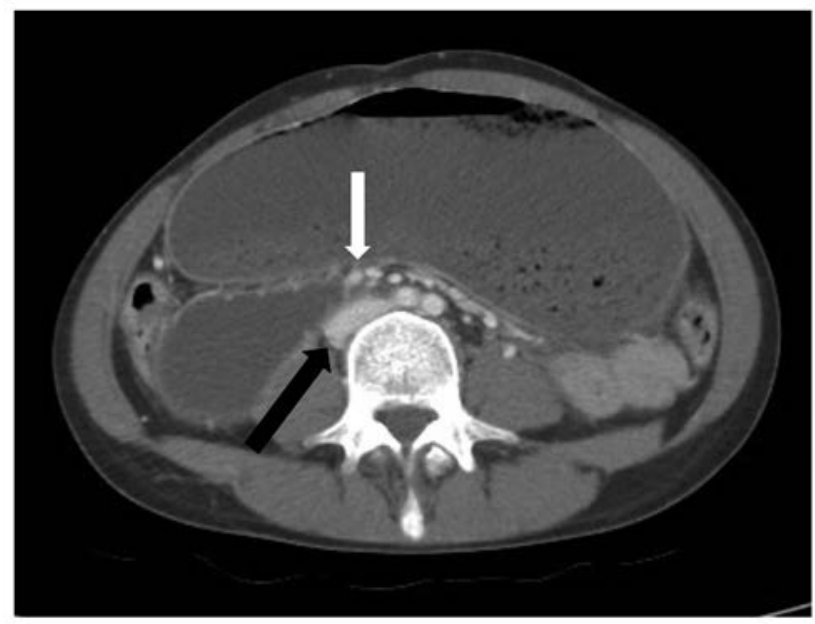

Fig. 9 SMA-like syndrome with duodenal obstruction due to compression by the distal SMV (white arrow) and the IVC (black arrow). IVC, inferior vena cava; GI, gastrointestinal; SMA, superior mesenteric artery; SMV, superior mesenteric vein.

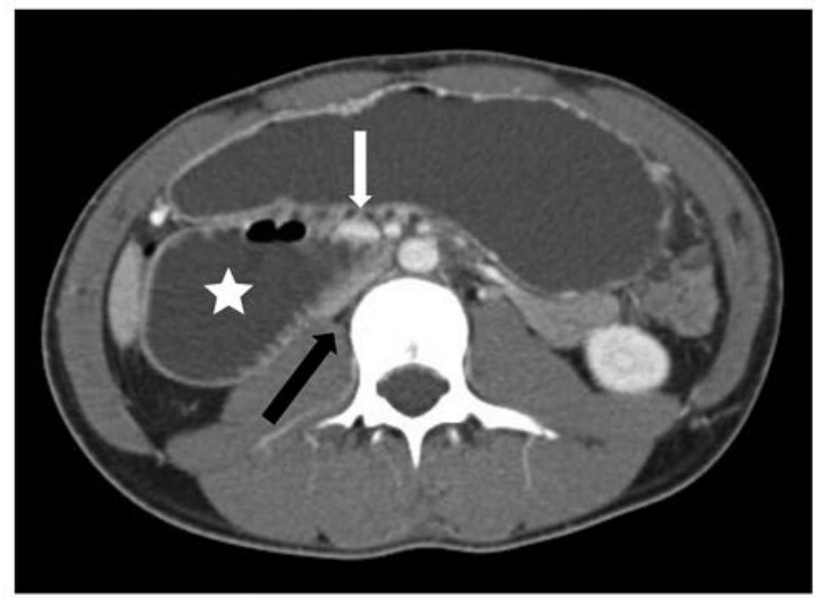

Fig. 10 (A) SMA-like syndrome with duodenal obstruction (white star) due to compression by the distal SMV (white arrow) and the collapsed IVC (black arrow). IVC, inferior vena cava; SMA, superior mesenteric artery; SMV, superior mesenteric vein.

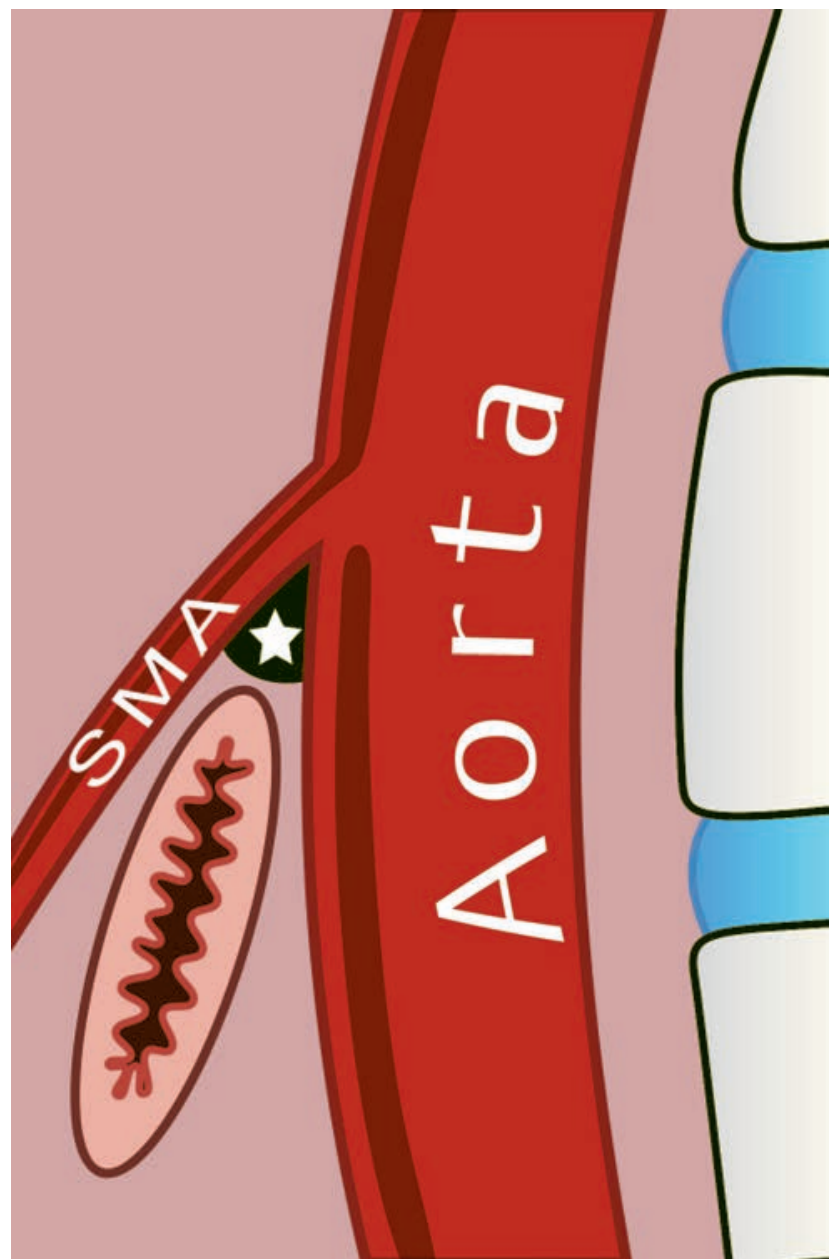

Fig. 11 The third portion of the duodenum, suspended by the ligament of Treitz, as it passes between the SMA and aorta. The acute downward angle of the SMA from the aorta (star) is exaggerated in classic SMAS, causing compression of the passing duodenum. SMA, superior mesenteric artery; SMAS, superior mesenteric artery syndrome.

Lamba et $\mathrm{al}^{8}$ state that all other causes of duodenal obstruction (benign or malignant strictures, tumors, aneurysms, and pancreatitis) must be excluded before a diagnosis of SMAS is made and includes only those cases where duodenal obstruction is due to compression between the SMA and abdominal aorta. Our series of cases, however, illustrate vascular duodenal compression disorders (with one exception) caused by highly unusual vascular compression other than the SMA and aorta classic for SMAS. Numerous mesenteric vessels course anterior to the third portion of the duodenum, as well as the large retroperitoneal vessels and their branches posteriorly ( - Fig. 12), which are potential sources of extrinsic compression. Additionally, variation in the length of the duodenal suspensory ligament can displace the duodenum inferiorly and to a position susceptible to compression by the cases in this series. Our series demonstrates, these compression syndromes may be caused by either arterial ( - Fig. 13A) and venous vasculature ( - Fig. 13B).

Several of the presented cases in this series present vascular duodenal compressive disorders, which to the best of 


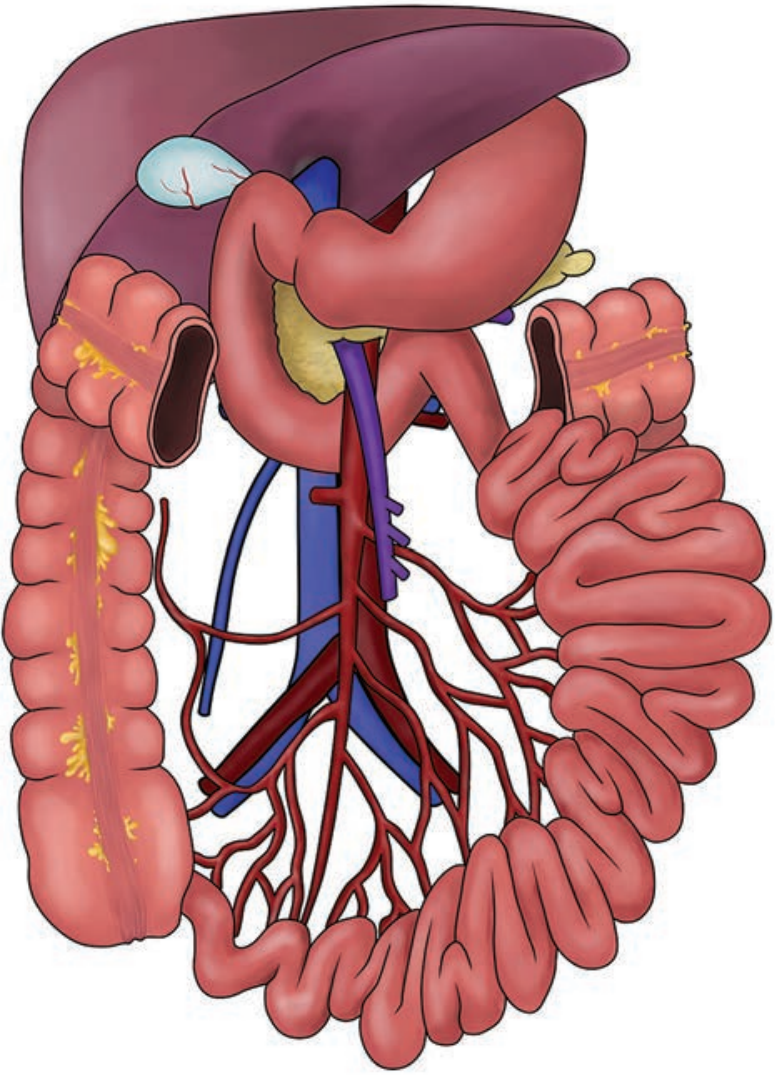

Fig. 12 Anatomic diagram detailing the major abdominal vasculature, which may contribute to a SMA-like syndrome. Note the SMA and SMV and their tributaries coursing anterior to the third portion of the duodenum. Note the aorta and IVC and their branches posteriorly in the retroperitoneum. In the setting of a low-hanging duodenal suspensory ligament, there are a greater number of branching vessels to potentially contribute to compression of the duodenum. IVC, inferior vena cava; SMA, superior mesenteric artery; SMV, superior mesenteric vein.

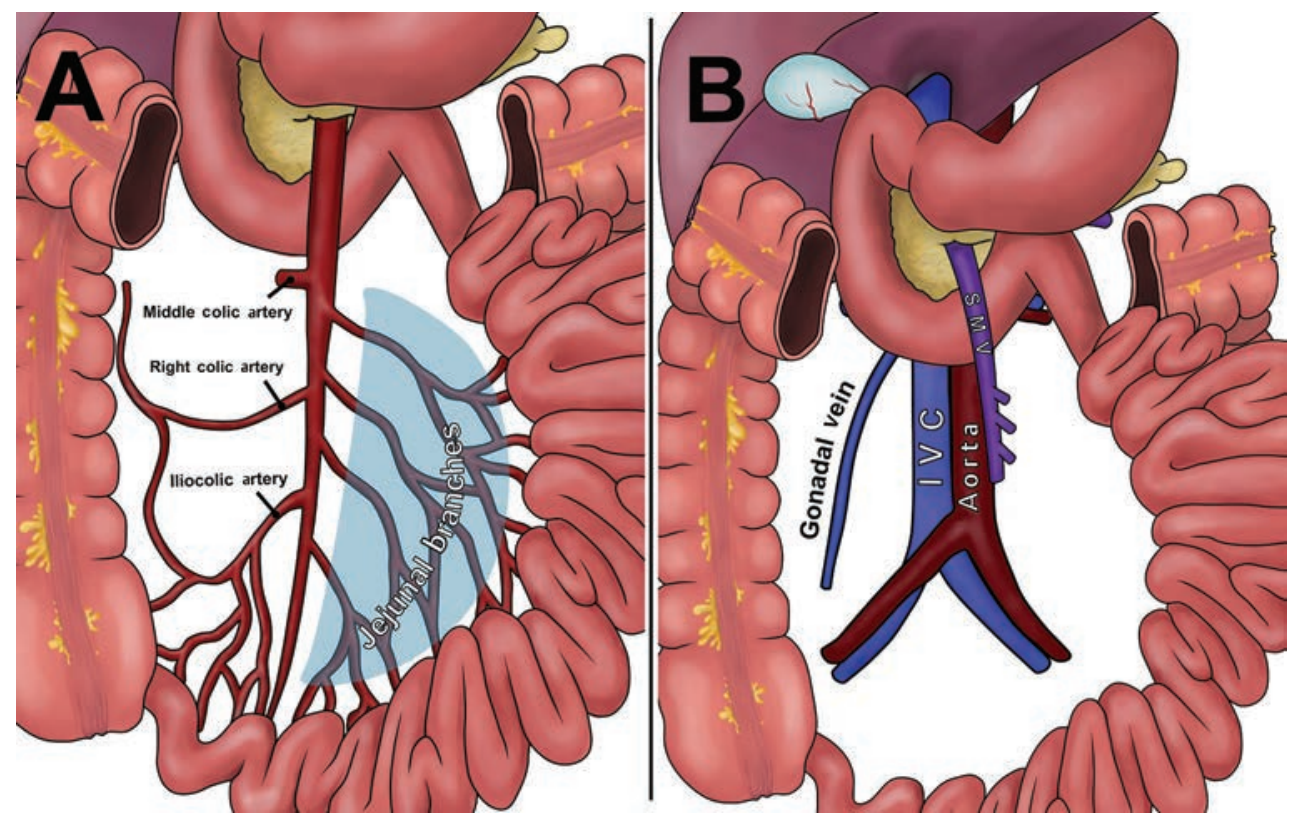

Fig. 13 Labeled anatomic diagram of the major abdominal vasculature. (A) The SMA emerges from behind the neck of the pancreas and crosses anterior to the third portion of the duodenum. The first major branch is the middle colic artery follow by multiple jujenal branches, the right colic artery, and the ileocolic artery. (B) The aorta and the IVC reside behind the duodenum in the retroperitoneum. The SMV courses anterior to the duodenum and behind the pancreas, converging with the splenic vein to form the portal vein. The right gonadal vein originates just proximal to the right renal vein. IVC, inferior vena cava; SMA, superior mesenteric artery; SMV, superior mesenteric vein. 
our knowledge, have never been reported before in the world literature. These include duodenal compression between the right gonadal vein and ileocolic vein (-Fig. 5); distal SMA branches and the aorta (including an abdominal aortic aneurism); SMV and aorta; SMA and left common iliac artery; ileocolic vein and IVC; distal branches of the SMV and IVC; as well as compression between the SMA/SMV and a retroperitoneal cyst. Shin et al ${ }^{16}$ use the terminology "SMA-like syndrome" in a patient who presented with duodenal obstruction in a patient demonstrating a variant SMV drainage consisting of dual intestinal venous trunks, with a dominant right trunk draining into a confluence dorsal to the SMA and directly over the third duodenal segment. An additional case of SMA-like syndrome was reported by Dinan et $\mathrm{a}^{17}$ in case of a normal anatomic variant replaced by right hepatic artery causing duodenal obstruction.

\section{Conclusion}

The diagnostic criterion for the diagnosis of the SMAS has been well established in the literature. We report a series of nine cases of unusual etiologies for vascular duodenal compression that do not meet the diagnostic criteria for the SMAS and propose that "SMA-like syndrome" be used to classify these unusual vascular duodenal compression cases.

\section{Conflict of Interest}

None declared.

\section{References}

1 Rokitansky CV, Lehrbuch Der Pathologischen Anatomie. Vienna: Braumüller \& Seidel; 1861;67

2 Wilkie D. Chronic duodenal ileus. Am. J Med Sci 1927;173(5):643-648

3 Gustafsson L, Falk A, Lukes PJ, Gamklou R. Diagnosis and treatment of superior mesenteric artery syndrome. Br J Surg 1984;71(7):499-501
4 Konen E, Amitai M, Apter S, et al. CT angiography of superior mesenteric artery syndrome. Am J Roentgenol 1998;171(5):1279-1281

5 Hines JR, Gore RM, Ballantyne GH. Superior mesenteric artery syndrome. Diagnostic criteria and therapeutic approaches. Am J Surg 1984;148(5):630-632

6 Applegate GR, Cohen AJ. Dynamic CT in superior mesenteric artery syndrome. J Comput Assist Tomogr 1988;12(6): 976-980

7 Unal B, Aktaş A, Kemal G, et al. Superior mesenteric artery syndrome: CT and ultrasonography findings. Diagn Interv Radiol 2005;11(2):90-95

8 Lamba R, Tanner DT, Sekhon S, McGahan JP, Corwin MT, Lall CG. Multidetector CT of vascular compression syndromes in the abdomen and pelvis. Radiographics 2014; 34(1):93-115

9 Merrett ND, Wilson RB, Cosman P, Biankin AV. Superior mesenteric artery syndrome: diagnosis and treatment strategies. J Gastrointest Surg 2009;13(2):287-292

10 Lee CS, Mangla JC. Superior mesenteric artery compression syndrome. Am J Gastroenterol 1978;70(2):141-150

11 Anderson JR, Earnshaw PM, Fraser GM. Extrinsic compression of the third part of the duodenum. Clin Radiol 1982;33(1):75-81

12 Welsch T, Büchler MW, Kienle P. Recalling superior mesenteric artery syndrome. Dig Surg 2007;24(3):149-156

13 Ahmed AR, Taylor I. Superior mesenteric artery syndrome. Postgrad Med J 1997;73(866):776-778

14 Gondos B. Duodenal compression defect and the "superior mesenteric artery syndrome” 1 . Radiology 1977;123(3):575-580

15 Cohen LB, Field SP, Sachar DB. The superior mesenteric artery syndrome. The disease that isn't, or is it? J Clin Gastroenterol 1985;7(2):113-116

16 Shin J, Shin PJ, Bartolotta RJ. SMA-like syndrome with variant mesenteric venous anatomy. Clin Imaging 2018;48:86-89

17 Dinan D, Epelman M, Guimaraes CV, Burns RC, Donnelly LF. Proximal duodenal obstruction associated with compression from a replaced right hepatic artery. Pediatr Radiol 2014;44(2):226-229 